\title{
iPSC Modeling of Presenilin1 Mutation in Alzheimer's Disease with Cerebellar Ataxia
}

\author{
Ling $\mathrm{Li}^{1 \dagger}$, Jee Hoon $\mathrm{Roh}^{2 \dagger}$, Eun Hyuk Chang ${ }^{3,4}$, Yoonkyung Lee ${ }^{1}$, Suji Lee ${ }^{1}$, \\ Minchul Kim ${ }^{1}$, Wonyoung Koh ${ }^{1}$, Jong Wook Chang ${ }^{3,4}$, Hee Jin Kim ${ }^{3,5}$, \\ Mahito Nakanishi ${ }^{6}$, Roger A. Barker ${ }^{7}$, Duk L. Na ${ }^{3,5 *}$ and Jihwan Song ${ }^{1 *}$
}

${ }^{1}$ CHA Stem Cell Institute, Department of Biomedical Science, CHA University, Seongnam 13488, ${ }^{2}$ Department of Neurology, Asan Medical Center, University of Ulsan College of Medicine, Seoul 05505, ${ }^{3}$ Neuroscience Center, Samsung Medical Center, Seoul 06351, ${ }^{4}$ Stem Cell \& Regenerative Medicine Institute, Samsung Medical Center, Seoul 06351, ${ }^{5}$ Department of Neurology, Samsung Medical Center, Seoul 06351, Korea, ${ }^{6}$ Research Center for Stem Cell Engineering, National Institute of Advanced Industrial Science and Technology (AIST), Ibaraki 305-8561, Japan, ${ }^{7}$ John van Geest Cambridge Centre for Brain Repair, Department of Clinical Neuroscience, University of Cambridge, Cambridge CB2 OPY, UK

Disease modeling of Alzheimer's disease (AD) has been hampered by the lack of suitable cellular models while animal models are mainly based on the overexpression of AD-related genes which often results in an overemphasis of certain pathways and is also confounded by aging. In this study, we therefore developed and used induced pluripotent stem cell (iPSC) lines from a middle-aged AD patient with a known presenilin 1 (PSEN1) mutation (Glu120Lys; PS1-E120K) and as a control, an elderly normal subject. Using this approach, we demonstrated that the extracellular accumulation of A $\beta$ was dramatically increased in PS1-E120K iPSC-derived neurons compared with the control iPSC line. PS1-E120K iPSC-derived neurons also exhibited high levels of phosphorylated tau, as well as mitochondrial abnormalities and defective autophagy. Given that the effect of aging is lost with iPSC generation, these abnormal cellular features are therefore indicative of PSEN1-associated AD pathogenesis rather than primary changes associated with aging. Taken together, this iPSC-based approach of AD modeling can now be used to better understand AD pathogenesis as well as a tool for drug discovery.

Key words: Alzheimer disease, stem cell, Presenilin1, Amyloid beta, Tau, Autophagy

Received September 10,2018, Revised October 18,2018, Accepted October 23, 2018

* To whom correspondence should be addressed.

Jihwan Song, TEL: 82-31-881-7140, FAX: 82-31-881-7249

e-mail: jsong5873@gmail.com

Duk L. Na, TEL: 82-2-3410-3591, FAX: 82-2-3410-0052

e-mail:dukna@naver.com

These authors contributed equally to the work.

\section{INTRODUCTION}

To date, the great majority of disease modifying clinical trials in Alzheimer's disease (AD) have not succeeded despite promising preclinical data. The potential reasons for this failure is that; (1) the agents so trialled are aimed at the wrong pathological target; (2) there is a loss of target engagement; (3) the treatment is given too late in the disease course; or (4) the previous models are affected by the effects of aging beyond $\mathrm{AD}$ pathogenesis $[1,2]$.

Conventional AD models are mainly based on the overexpres- 
sion of $\mathrm{AD}$-related genes which overemphasizes by its very nature certain pathogenic pathways, thereby limiting the clinical translation of such approaches. In particular, the most popular mouse models of $\mathrm{AD}$ are based on the overexpression of each or combinations of amyloid precursor protein (APP), Presenilin 1 (PSEN1), or Presenilin 2 (PSEN2), all of which result in amyloid beta (A $\beta$ ) plaques and inflamation. However, these animals rarely show any neurodegeneration in the brain by the time of death $[3,4]$. On the other hand, AD patients are known to have a very long preclinical phase to their manifest illness which can exceed 10 20 years, during which time aging is known to affect $\mathrm{AD}$ pathophysiology and the subsequent neurodegernation. Therefore, to eliminate or minimize the effect of aging, we focused on autosomal dominant $\mathrm{AD}(\mathrm{ADAD})$ patients, who develop $\mathrm{AD}$ symptoms in middle age. In addition, we employed an induced pluripotent stem cell (iPSC)based technology to model AD pathophysiology, in which the effect of aging is not normally reflected during reprogramming process [5]. To do this, we generated iPSC lines from an AD patient with a known PSEN1 mutation (Glu120Lys; PS1-E120K) and from an elderly normal subject, as a control. The main purpose of this study was to investigate whether the PS1-E120K iPSC-derived neurons manifest the cardinal pathological features of $\mathrm{AD}$, including aggregation of $A \beta$ and tau. Given that the effect of aging is not normally refected in iPSCs, we anticipated that the iPSC model of this $\mathrm{AD}$ patient would better reveal the pathogenic conesequences of a PS1-E120K mutation, with no or only limited effects from aging.

Using this approcah, we have demonstrated that the level of extracellular deposition of A $\beta$ dramatically increased in PS1-E120K iPSC-derived neurons, compared with the control iPSC-derived neurons. Furthermore, we showed that PS1-E120K iPSC-derived neurons expressed high levels of phosphorylated tau along with abnormal mitochondrial activity and defective autophagy.

\section{MATERIALS AND METHODS}

\section{Enrollment of participants}

The patient met the criteria for $\mathrm{AD}$ as recommended by the National Institute on Aging-Alzheimer's Association [6] and the normal elderly subject fulfilled the criteria of a normal elderly control as defined by Christensen [7]. We performed detailed neuropsychological tests, MRI, [18F]-Florbetaben or [11C]-Pittsburgh compound B (PIB) amyloid PET scans, and blood draw for iPSC generation from each participant. The Institutional review board of CHA University (1044308-201612-BR-031-02), Samsung Medical Center (2013-03-087), and Asan Medical Center (20150699) approved the study protocol, and informed written consent was obtained from each participant. All methods were performed in accordance with the relevant guidelines and regulations.

\section{Generation of iPSC lines}

Mononuclear cells (MNCs) were isolated freshly from the peripheral blood of the $\mathrm{AD}$ patient and normal subject using the Ficoll-Paque ${ }^{\mathrm{TM}}$ PLUS method (GE Healthcare, USA). Isolated peripheral-derived MNCs (PBMCs) were cultured for 4 days in MNC media containing $50 \mathrm{ng} / \mathrm{ml}$ interleukin-6 (IL-6), $50 \mathrm{ng} / \mathrm{ml}$ stem cell factor (SCF), $10 \mathrm{ng} / \mathrm{ml}$ thrombopoietin (TPO), $20 \mathrm{ng} / \mathrm{ml}$ Flt3 ligand (Flt-3L), $20 \mathrm{ng} / \mathrm{ml}$ interleukin-3 (IL-3), and $10 \mathrm{ng} / \mathrm{ml}$ granulocyte colony-stimulating factor (G-CSF) (all from WAKO, Japan) in StemFit AK03 medium (kindly provided by Ajinomoto, Japan). The MNCs were then infected with SeVdp (KOSM) 302L at MOI of 3 10 [8] and transferred into 6-well dishes coated with iMatrix-511 (Matrixome, Japan). From the next day, 500 ul of StemFit AK03 medium was added every day for 4 days, after which the medium was fully changed every other day until iPSClike colonies emerged. Sub-culturing and expansion of the cells was then undertaken until the generated iPSCs became stable for characterization and storage, normally at passage 10 .

\section{Karyotyping and genotyping}

Karyotyping was performed using a GTG-banding analysis (Korea Research of Animal Chromosomes, Korea). The iPSC pellets were incubated in lysis buffer containing $100 \mathrm{mM}$ Tris- $\mathrm{HCl}(\mathrm{pH}$ 8.0), $50 \mathrm{mM}$ EDTA, $0.2 \%$ SDS, $200 \mathrm{mM}$ NACL and $200 \mathrm{ug} / \mathrm{ml}$ Proteinase $\mathrm{K}$ for $3 \mathrm{hr}$ at $55^{\circ} \mathrm{C}$. Genomic DNA was isolated by isopropyl alcohol precipitation, followed by a 70\% ethanol washing step. Genotyping of the PS1-E120K single nucleotide mutation was performed by PCR amplification of isolated iPSC genomic DNA, followed by DNA sequencing (Cosmo Genetech, Korea). The PS1 gene was amplified by PCR using the following primers (forward primer: GTA GAA TCT ATA CCC CAT TC; reverse primer: TCA CCT TAT AGC ACC TGT AT).

\section{Reverse transcription- polymerase chain reaction (RT-PCR) and Quantitative-PCR (Q-PCR)}

Total RNAs were manually isolated using the TRIzol reagent (Life Technologies) lysis and isopropyl alcohol precipitation. Complementary DNAs (cDNAs) were synthesized using the cDNA synthesis Kit (Cosmo Genetech, Korea). RT-PCR amplification was done in a final volume of $20 \mathrm{ul}$ containing $200 \mathrm{ng} / \mathrm{ul} \mathrm{cDNA}$ for each sample. Primers were used as follows: GAPDH (forward primer: TGA CCA CAG TCC ATG CCA TCA CTG C; reverse primer: GTC ATA CCA GGA AAT GAG CTT GAC A); OCT4 (forward primer: CTG AAG CAG AAG AGG ATC AC; reverse 
primer: GAC CAC ATC CTT CTC GAG CC); NANOG (forward primer: TTC TTG ACT GGG ACC TTG TC; reverse primer: GCT TGC CTT GCT TTG AAG CA); SOX2 (forward primer: GCT GCA AAA GAG AAC ACC AA; reverse primer: CTT CCT GCA AAG CTC CTA CC); LIN28 (forward primer: CAC CAT GGG CTC CGT GTC CAA CCA GCA G; reverse primer: TCA ATT CTG TGC CTC CGG GAG CAG GGT AGG). Q-PCR analysis was performed using TB Green ${ }^{\mathrm{TM}}$ Premix Ex Taq ${ }^{\mathrm{TM}}$ (TaKaRa) with $100 \mathrm{ng} / \mathrm{ul}$ cDNA for each sample. Primers were used as follows: PSEN1 (forward primer: ACA GAG TTA CCT GCA CCG TTG T; reverse primer: GCT ATA AGG TCA TCC ATG CCT G); DRP1 (forward primer: ATG TTG CAT CTG GAG GTG GT; reverse primer: CGT GAA CCT GCT AGA TGT GC); Fis1 (forward primer: CAG TTT GAG TAC GCC TGG TG; reverse primer: CCG GCT CAA GGA ATA CGA GA); Mfn1 (forward primer: CTG GCT GTC TTG TAC GTG TG; reverse primer: CAA GGT GAA TGA GCG GCT TT); Mfn2 (forward primer: GAC CCC GTT ACC ACA GAA GA; reverse primer: GCT CTG GGA CAA AGT TCT GC).

\section{Sendai virus detection PCR}

After total RNA isolation and cDNA synthesis, Sendai virus detection RT-PCR was performed using a final volume of $20 \mathrm{ul}$ with a $200 \mathrm{ng} / \mathrm{ul}$ concentration for each sample. Primers were used as follows: SeVdp NP (forward primer: AGA CCC TAA GAG GAC GAA GAC AGA; reverse primer: ACT CCC ATG GCG TAA CTC CAT AG) for detecting the nucleoprotein (NP) of Sendai virus vector (SeVdp) [9].

\section{Mycoplasma detection PCR}

After genomic DNA isolation as described above, we detected the mycoplasma contamination in cell culture using the PCRbased e-Myco ${ }^{\mathrm{TM}}$ VALiD Mycoplasma detection Kit (iNtRON, Korea). PCR was done using a final volume of $20 \mathrm{ul}$ containing 100 ng/ul of each sample $[9,10]$.

\section{In Vitro differentiation}

Human iPSCs were harvested using a 0.5\% TrypLE Select (ThermoFisher) treatment and used for embryoid body (EB) formation. Detached cells were transferred into 96-well Nunclon ${ }^{\mathrm{TM}}$ Sphera $^{\mathrm{TM}}$ Microplate (ThermoFisher) that includes DMEM/F12 medium containing 20\% Knockout serum replacement (KSR; Life Technology), $0.1 \mathrm{M}$ nonessential amino acids (NEAA), 0.1 M 2-mercaptoethanol (Life Technologies) and 0.1 antibiotic-antimycotic solutions (ThermoFisher). The medium was changed every other day and the EBs were plated onto $0.2 \%$ gelatin-coated glass coverslips at day 8. EBs were then allowed to differentiate spontaneously for an additional 8 days.

\section{Cortical neuronal differentiation}

For the cortical neuronal differentiation, iPSCs were dissociated into single cells and quickly re-aggregated in a 96-well Nunclon ${ }^{\text {TM }}$ Sphera ${ }^{\text {TM }}$ Microplate (ThermoFisher). The EBs were cultured in DMEM/F12 containing 20\% knockout serum replacement (KSR), 0.1 M nonessential amino acids (NEAA), 0.1 M 2-mercaptoethanol, $0.1 \%$ antibiotic-antimycotic solutions (all from ThermoFisher), 10 uM SB431542 (Reagent Direct) and 10 uM LDN 193189 (Reagent Direct) for neural induction for 8 days. These neural induced EBs were dissociated using Accutase ${ }^{\mathrm{TM}}$ (Stem Cell Technologies) and were seeded onto $15 \mathrm{ug} / \mathrm{ml}$ poly-L-ornithine (Sigma) and $5 \mathrm{ug} / \mathrm{ml}$ laminin (Sigma)-coated dishes. Once neural precursor cells (NPCs) were formed, they were cultured in DMEM/F12 media containing $0.1 \mathrm{M}$ nonessential amino acids (NEAA), $0.1 \mathrm{M}$ 2-mercaptoethanol, 0.1 antibiotic-antimycotic solutions, $1 \% \mathrm{D}$ Glucose (Life Technologies), 1\% B27 supplement without vitamin A (all from ThermoFisher), $200 \mathrm{mM}$ L-Glutamine (Sigma), and 20 $\mathrm{ng} / \mathrm{ml}$ basic fibroblast growth factor (bFGF; PeproTech Korea). Finally, NPCs were differentiated into cortical neurons for 10 weeks in Neurobasal A medium containing 1\% B27 supplement without vitamin A, 1x Glutamax (all from ThermoFisher), $10 \mathrm{ng} / \mathrm{ml}$ brainderived neurotrophic factor (BDNF), $10 \mathrm{ng} / \mathrm{ml}$ glial cell-derived neurotrophic factor (GDNF), $10 \mathrm{ng} / \mathrm{ml}$ nurotrophin-3 (NT3) (all from PeproTech Korea) on PLO/laminin-coated dishes [10].

\section{Immunocytochemistry}

The cells were fixed with 4\% paraformaldehyde (PFA) for 15 min at room temperature (RT). Fixed cells were permeabilized and washed with TPBS containing $0.1 \%$ TritonX-100 (Sigma) in phosphate-buffered saline (PBS) and blocked with 5\% normal horse serum (Vector Labs) for 30 min at RT. Afterwards, they were incubated with primary antibodies in blocking solution overnight with gentle rocking at $4^{\circ} \mathrm{C}$. After washing three times with TPBS, they were incubated with secondary antibodies (ThermoFisher) for $90 \mathrm{~min}$ at RT, and then were counterstained using DAPI for $15 \mathrm{~min}$. All fluorescence images were captured by confocal microscopy (TCSSP5II, Leica). The following primary antibodies were used: anti-OCT4 (1:200, Santa Cruz), anti-SOX2 (1:200, Millipore), anti-NANOG (1:200, R\&D Systems), anti-SSEA-4 (1:100, Developmental Studies Hybridoma Bank)), anti-TRA-1-81 (1:100, CHEMICON), Tuj1 anti-tubulin beta III isoform (1:200, Millipore), anti-SMA (1:100, DAKO); anti-AFP (1:100, DAKO), antiNestin (1:200, R\&D Systems), anti-Musashi (1:200, Millipore), anti-Map2 (1:200, Millipore), anti-TBR1 (1:100, Abcam), anti-CTIP2 (1:100, Abcam), 6E10 anti-Amyloid $\beta$ (1:500, Covance), AT8 anti- 
p-tau (1:1000, ThermoFisher), anti-ChAT (1:200, Millipore) and anti-LC3B (1:500, Cell Signaling).

\section{Extracellular and intracellular amyloid- $\beta$ ELISA}

Conditioned media (CM) were collected from cultured neuronal cells $\left(1 \times 10^{5}\right)$ at 48 hours after the last medium change from 6 and 10 weeks of differentiation. The pellets of iPSC-derived neurons were resuspended in RIPA buffer (Biosesang) containing protease inhibitors (ThermoFisher) and phosphatase inhibitors (ThermoFisher). Then they were chilled on ice for $30 \mathrm{~min}$ and sonicated. The supernatants were collected after centrifugation at 13,000 rpm for $30 \mathrm{~min}$ to remove membrane lipids. Protein concentration was determined using a BCA Protein Assay Kit (ThermoFisher). Intracellular $A \beta_{42}$ and $A \beta_{40}$ were measured in a total of $1 \mu \mathrm{g}$ proteins from 10 week-differentiated neurons. $A \beta_{40}$ and $A \beta_{42}$ levels were measured using the human $A \beta_{40}$ and $A \beta_{42}$ ELISA Kit according to the manufacture's instruction (IBL). ELISA plate reader (BioTek) was used to quantify $A \beta_{40}$ and $A \beta_{42}$ levels.

\section{Western blot analysis}

Cultured neurons in 6-well plates were harvested at 10 weeks of differentiation as cell pellets and were resuspended in RIPA buffer (BIOSESANG) containing protease inhibitors (ThermoFisher) and phosphatase inhibitors (ThermoFisher). They were then chilled on ice for $30 \mathrm{~min}$ and sonicated. The supernatants were collected after centrifugation at 13,000 rpm for $30 \mathrm{~min}$ to remove membrane lipids. Protein concentration was determined using a BCA Protein Assay Kit (ThermoFisher), and 25 ug of protein for each sample was electrophoresed on $8 \%$ or $12 \%$ gels. The separated samples were transferred onto a PVDF membrane and incubated with target antibodies. Protein bands were visualized by Immobilon Western (Millipore) and were detected using Chemi-Doc (BioRad). The following primary antibodies were used: Tau 5 anti-tau (1:1,000, ThermoFisher), AT8 anti-p-tau (1:1,000, ThermoFisher), anti-OPA1 (1:1,000, Santa Cruz), anti-Mfn 1 (1:1,000, Abcam), anti-Mfn2 (1:1,000, Cell Signaling), anti-Drp1 (1:1,000, Cell Signaling), anti-Fis1 (1:1,000, Santa Cruz), anti-Ub (1:4,000, Santa Cruz), anti-LC3B (1:1,000, Cell Signaling), anti-LAMP2 (1:1,000, Santa Cruz), anti-Beclin 1 (1:1,000, Cell Signaling), p62 anti-SQSTM1 (1:1,000, Santa Cruz), 4G8 anti-APP (1:500, Covance), anti-pDrp1 (Ser637, 1:1000, Cell Signaling) and anti- $\beta$-actin (1:10000, Santa Cruz).

\section{Autophagy flux assay and Cyto ID live cell staining}

For autophagy flux assay, we treated cells with $10 \mathrm{uM}$ chloroquine (CQ) for $12 \mathrm{~h}$, a compound known to interference the lysosomal function [11]. After $12 \mathrm{~h}$, we used the CytoID ${ }^{\circledast}$ Green Autophagy
Detection Kit (Enzo Life Sciences Inc., Cat. No, ENZ-51031-K200) to detect the autophagic vesicles in live cells. Induction of autophagic flux can be visualized by enhanced accumulation of autophagic vesicles when lysosomal function is inhibited. CytoID green was co-stained with DAPI and the fluorescence images were captured using a confocal microscopy (TCSSP5II, Leica). The quantification for the number and size of autophgic vesicles were performed using Image J (particle analysis plugin).

\section{Statistical analysis}

All statistical analyses were performed using a one-factor analysis of variance (ANOVA) followed by a Fisher's LSD (Least Significant Difference) using the Statistical Analysis System (Enterprise 4.1, SAS Korea, Seoul, Korea). Significance was accepted at the 95\% probability level. Data in graphs are presented as mean \pm SEM. pvalue $\left.<0.05{ }^{*}\right)$, p-value $\left.<0.01{ }^{(* *}\right)$ and p-value $\left.<0.001{ }^{(* *}\right)$.

\section{RESULTS}

\section{Clinical characteristics of the patient}

The patient was a 38-year-old woman who visited the outpatient clinic of Samsung Medical Center, Seoul, Korea. She had progressive memory impairment over a course of five years, which eventually resulted in disturbance of her daily life. She had 16 years of education and had no history of medical illness. A mildly spastic gait was noticed by her husband 6 months prior to presentation. Neurological examination revealed cerebellar dysfunction, including limb and truncal ataxia, dysdiadochokinesia, cerebellar dysarthria, and ocular abnormalities, such as overshooting saccades and

Table 1. Neuropsychological test results of the patient

\begin{tabular}{lcc}
\hline & Raw score & Percentile score \\
\hline Digit span Forward & $9 / 9$ & 95.82 \\
Digit span Backward & $4 / 8$ & 59.87 \\
K-BNT & $38 / 60$ & 8.08 \\
RCFT Copy score & $29 / 36$ & $<0.01$ \\
SVLT Immediate recall & $11 / 36$ & 1.04 \\
SVLT Delayed recall & $0 / 12$ & 0.04 \\
SVLT Recognition & $11 / 24$ & $<0.01$ \\
RCFT Immediate recall & $2.5 / 36$ & 3.67 \\
RCFT Delayed recall & $2.5 / 36$ & 0.84 \\
RCFT Recognition & $13 / 24$ & 0.13 \\
COWAT Animal & $10 / 20$ & 0.62 \\
COWAT Supermarket & $10 / 20$ & 14.23 \\
COWAT Phonemic & $28 / 45$ & 87.29 \\
Stroop color reading & $81 / 112$ & 28.1 \\
\hline
\end{tabular}

K-BNT, Korean version of the Boston Naming Test; RCFT, Rey-Osterrieth Complex Figure Test; SVLT, Seoul Verbal Learning Test; COWAT, Controlled Oral Word Association Test. 
saccadic intrusions during smooth pursuit eye movements. She also had bilateral spasticity in her legs and bradykinesia in both upper extremities. She demonstrated global cognitive problems with deficits in verbal and visual memory, language, and visuospatial functions with relative sparing of attention and frontalexecutive function (Table 1).

The patient had a family history of dementia and ataxia (Fig. 1A). Her grandmother (I:2 in Fig. 1A) had developed a late-onset dementia during the seventh decade of life and died ten years after the diagnosis. Her cousin (III:2 in Fig. 1A) was diagnosed with late-onset cerebellar ataxia at the age of 54 and died at 62 years of age due to aspiration pneumonia without evidence of cognitive impairment. Brain MR images of her cousin (III:2) demonstrated severe cerebellar atrophy (Fig. 1B) without prominent atrophy of the medial temporal lobes. Brain MRI of our patient revealed generalized cortical atrophy along with atrophy in the bilateral medial temporal lobes and cerebellum (Fig. 1C). Laboratory studies of the patient, including 24-hour urine copper levels were unremarkable. Given the tentative diagnosis of dementia associated with a familial cerebellar ataxia, genetic evaluations for spinocerebellar ataxia (SCA, 1, 2, 3, 6, 7, 8, 12, 17, 27), dentatorubral-pallidoluysian atrophy (DRPLA), and late-onset Friedreich's ataxia (Frataxin gene) were performed and shown to be negative. Huntingtin gene test was negative. Given the diagnosis of early onset dementia with spastic paraparesis, a genetic work-up for a Presenilin mutation was performed [12], and a mutation in the PSEN1 gene (a transition from $\mathrm{G}\left(358^{\text {th }}\right.$ codon) to A, resulting in a change from glutamate to lysine at codon 120 (Glu120Lys; PS1-E120K)) was detected. For the control iPSC generation, blood samples were obtained from a cognitively normal elderly man aged 72 , who fulfilled the criteria for being a normal elderly person [7].

Pittsburgh compound-B ( $\mathrm{PiB}$ ) positron emission tomography (PET) imaging of the patient revealed a deposition of $A \beta$ in the striatum as well as in the bilateral frontal lobes, posterior cingulate cortices, and precuneus (Fig. 1D). These PiB-PET image findings of the patient are compatible with previous reports of familial AD with spastic paraparesis caused by PSEN1 mutations $[13,14]$. The PiB-PET images of our patient did not reveal amyloid deposition in the cerebellum as shown in some previous studies $[13,15]$. The MRI and ${ }^{18} \mathrm{~F}$-Florbetaben amyloid PET images of the normal elderly subject were in normal range.

\section{Generation of iPSC lines and cortical neuronal differentiation}

Isolated MNCs were reprogrammed using Sendai virus vector (SeVdp) which expresses four reprogramming factors (OCT3/4, SOX2, cMYC, and KLF4) [9]. In our iPSC generation procedure, we normally pick more than three individual clones and select the best growing clone among them for further analyses, including neuronal differentiation experiments. Although the effects of PS1-E120K mutation on $A \beta$ deposition have been studied in the brains of familial AD patients previously [16, 17], the pathological features of the patients are less known at cellular levels. For this reason, we generated an $\mathrm{PSC}$ line from AD patient carrying a mutation for PS1-E120K using iPSC technology for the first time. Control and PS1-E120K mutation iPSC lines exhibited the typical expression of undifferentiated pluripotent stem cell markers, such as OCT4, SOX2, SSEA4 and TRA-1-81 (Fig. 2A, B). Genotyping of the established iPSC lines was confirmed using a conventional sequencing method (Fig. 2C). The differentiation potential of iPSC lines was assessed in vitro by three-germ layer marker expression (Fig. 2D). Control and PS1-E120K mutation iPSC lines did not carry a $\mathrm{SeV}$ integration (Fig. 2E) and were not contaminated by mycoplasma (Fig. 2F, G).

To characterize the cortical neurons derived from each iPSC line, we developed a modified cortical neuron differentiation protocol, based on previously published procedures (Fig. 3A) [10]. Differentiated cells expressed general neuronal markers (i.e., Tuj1 and Map2), cholinergic marker (i.e., ChAT), as well as cortical neuron markers (i.e., TBR1 and CTIP2; Fig. 3B, C). We observed high levels of neuronal differentiation in our experiments, and the following is the relative percentage of each neuronal cell type (control vs. PS1-E120K iPSC-derived neurons): TUJ1 $(86.79 \% \pm 3.37$ vs. $82.41 \% \pm 4.42)$ MAP2 $(80.24 \% \pm 2.47$ vs. $80.45 \% \pm 1.81)$, TBR1 $(61.29 \% \pm 2.23$ vs. $64.69 \% \pm 3.47)$ and CTIP2 $(59.65 \% \pm 3.28$ vs. $49.40 \% \pm 6.73)$. Among them, the percentage of TBR1/CTIPdouble positive neurons were relatively high $(58.02 \% \pm 4.43 \mathrm{vs}$. $48.41 \% \pm 6.93$ ), indicating our cortical neuron differentiation was efficient. We also observed high levels of ChAT, a marker for cholinergic neurons ( $74.25 \% \pm 6.69$ vs. $81.20 \% \pm 2.23$ ). In most cases, no significant differences in the neuronal differentiation propensity was seen between control and PS1-E120K iPSC lines (Fig. 3D). In addition, there was no significant difference of PSEN1 gene expression in iPSC and iPSC-derived neurons (data not shown). Moreover, no significant difference in features associated with aging was observed between the two iPSC lines (i.e., PS1-E120K iPSC from the 38 year-old AD patient $v s$. control iPSC from the 72 year-old normal elderly subject).

\section{Detection of A $\beta$ secretion from the PS1-E120K iPSC-derived neurons}

To analyze the functional aspects of familial AD (PS1-E120K), we investigated $A \beta$ secretion in the conditioned medium (CM) from the iPSC-derived neurons at 6 weeks and 10 weeks after differentiation. We measured $A \beta_{40}$ and $A \beta_{42}$ levels $48 \mathrm{hr}$ after the last 
A
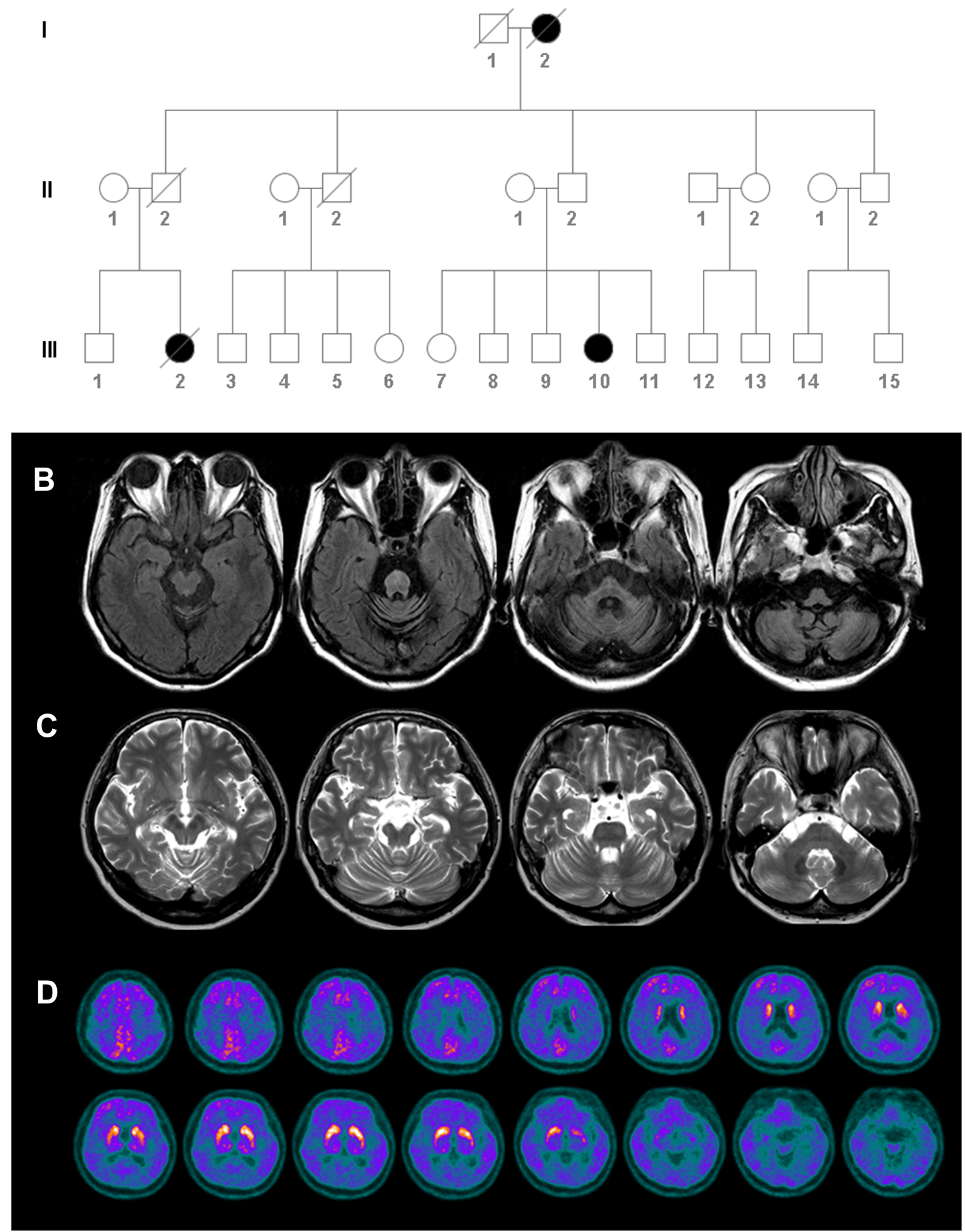

Fig. 1. Pedigree of the patient's family and brain MR images of the cousin of the patient and MR and Pittsburgh compound-B (PiB) positron emission tomography (PET) images of the patient. (A) Pedigree of the patient. I-2: grandmother of the patient, dementia; III-2: cousin of the patient, late onset cerebellar ataxia; III-10: the patient, dementia, spastic paraparesis, cerebellar ataxia. (B) Fluid attenuated inversion recovery (FLAIR) MR images of the cousin show severe ponto-medullo-cerebellar atrophy without atrophy in the medial temporal lobes. (C) T2-weighted axial MR images of the patient (III-10 in the pedigree) showing atrophy bilaterally of the medial temporal lobes and prominent volume loss in the cerebellum with enlargement of the fourth ventricle. (D) Increased amyloid deposition in the bilateral striatum, frontal and posterior cingulate cortices, and precuneus, but not in the cerebellum. 
A

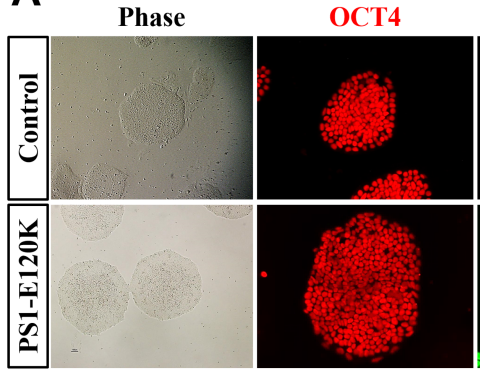

C

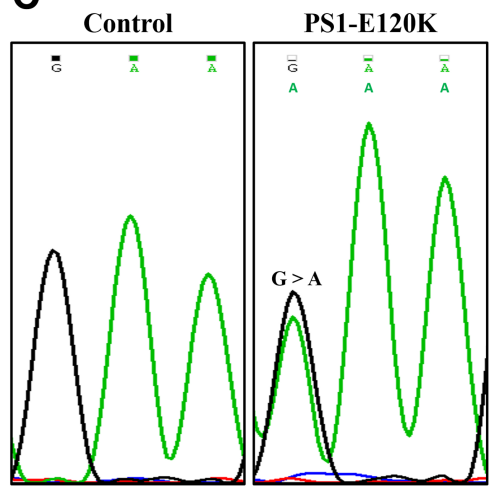

SOX2
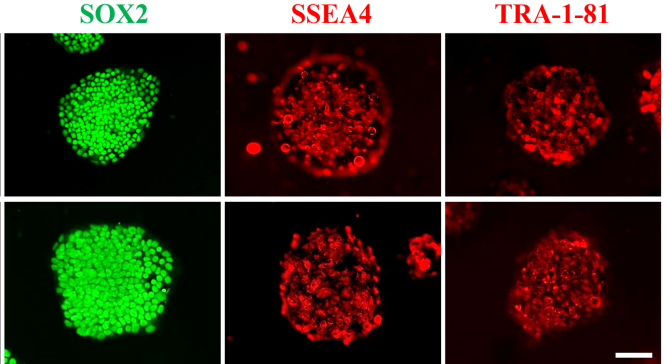

D

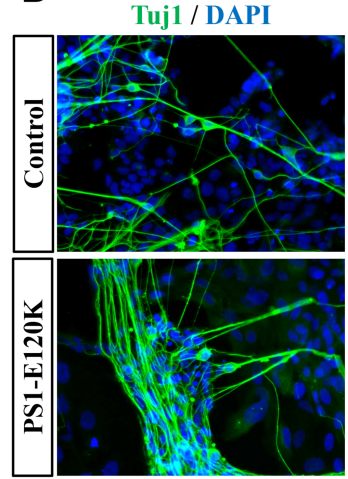

SMA / DAPI

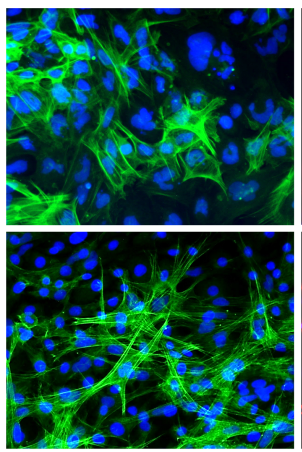

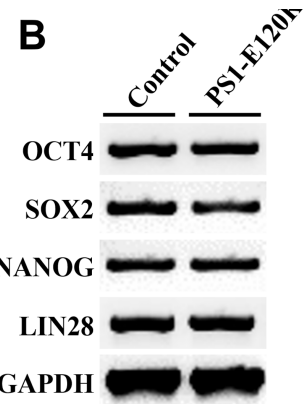

AFP / DAPI

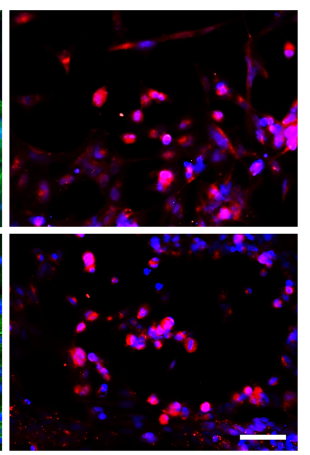

E

Control (46, XY)

PS1-E120K (46, XX)

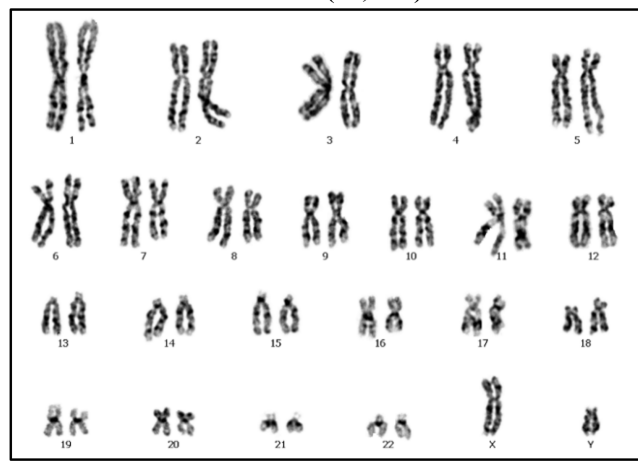

औ)

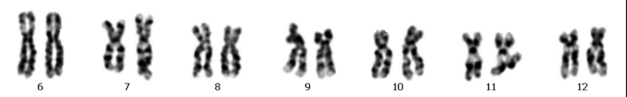

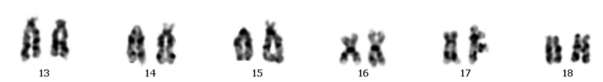

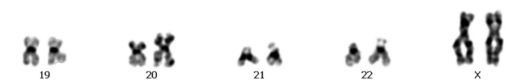
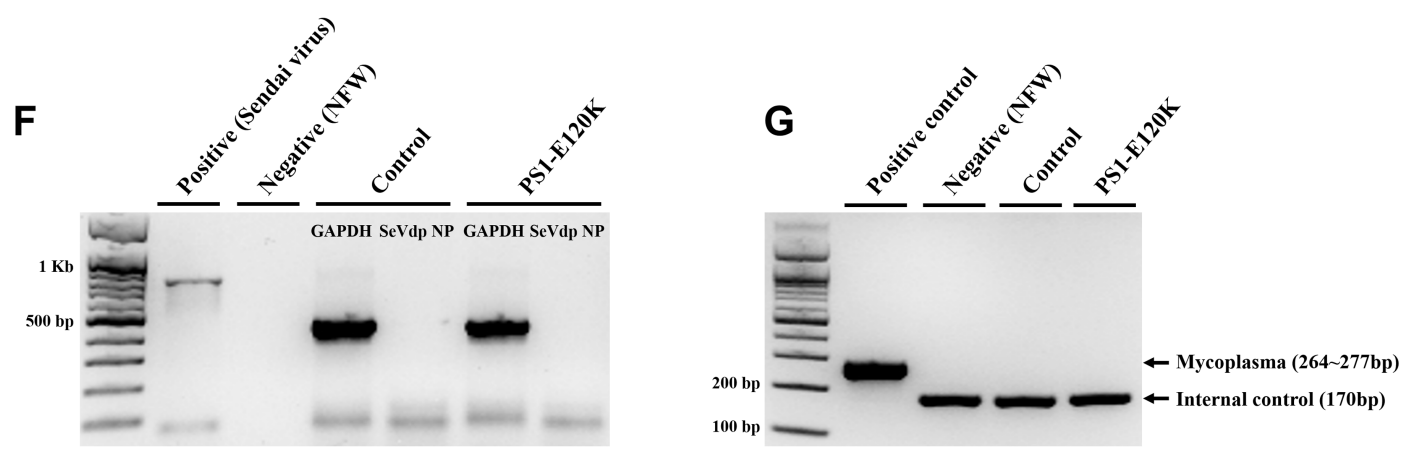

Fig. 2. Generation of iPSCs from an AD patient harboring a PSEN1 (E120K) mutation, and an eldely normal subject. (A) Established iPSC lines from both control and PS1-E120K patient showing the expression of pluripotent stem cell markers, such as OCT4 (red), SOX2 (green), SSEA4 (red) and TRA1-81 (red). (B) Reverse transcription PCR (RT-PCR) showing the expression of pluripotency markers (OCT4, SOX2, NANOG, SSEA4 AND TRA-181) in both iPSC lines. (C) Genomic DNA sequences showing the presence of the heterozygous E120K mutation (GAA to AAA) in the PSEN1 gene of the PS1-E120K -iPSC line. (D) Immunofluorescence analysis showing the potential of iPSC lines to form three germ layers, including ectoderm (type III $\beta$-tubulin [TUJ1], green), mesoderm (smooth muscle actin [SMA], green), and endoderm ( $\alpha$-fetoprotein [AFP], red). Scale bar: $100 \mu$ m. (E) Karyotype analysis of the control and PS1-E120K iPSC lines. (F) Reverse-transcription PCR analysis showing the absence of integration of the Sendai virus vectors. (G) PCR analysis showing no contamination by mycoplasma. 

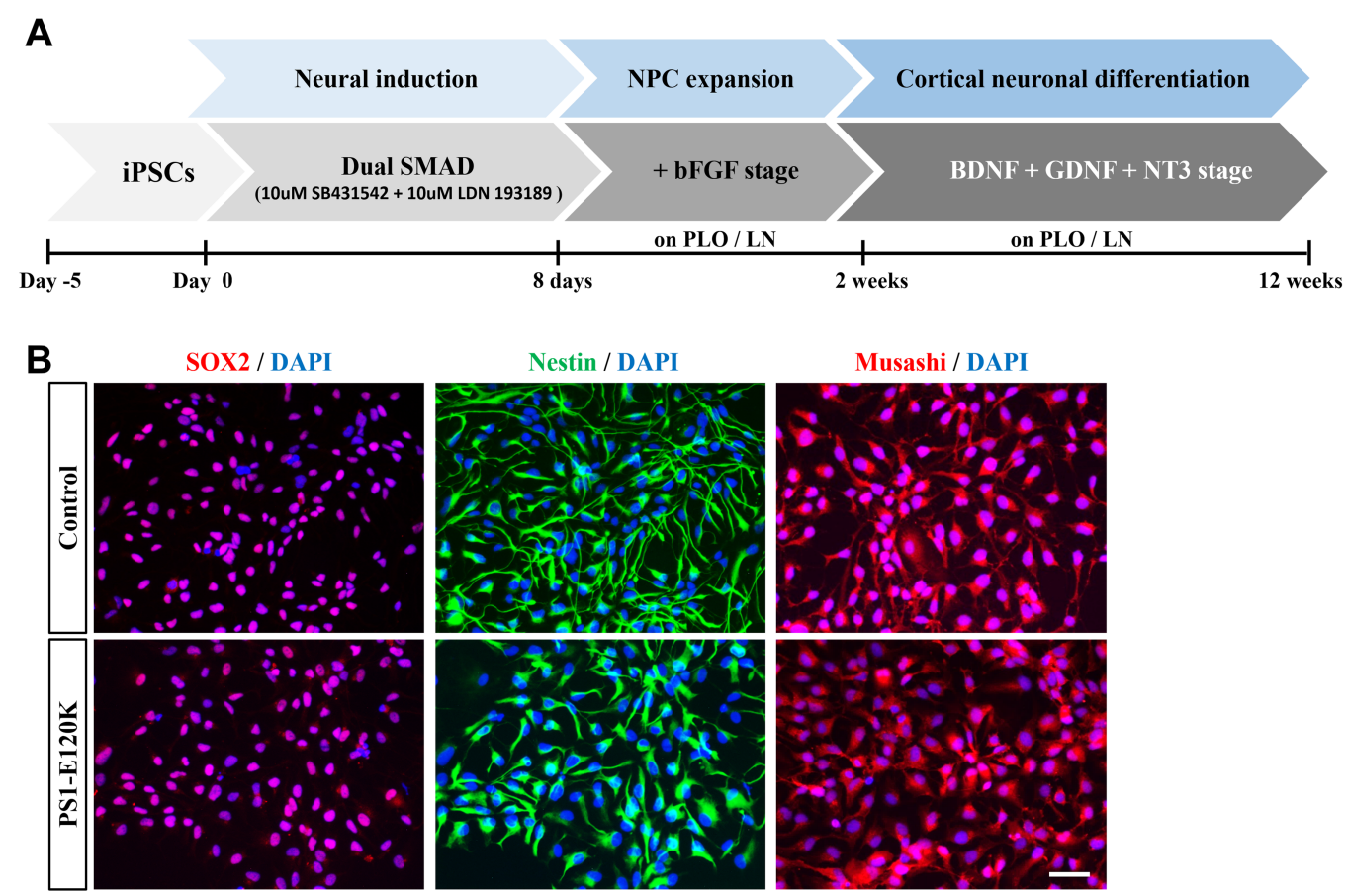

C
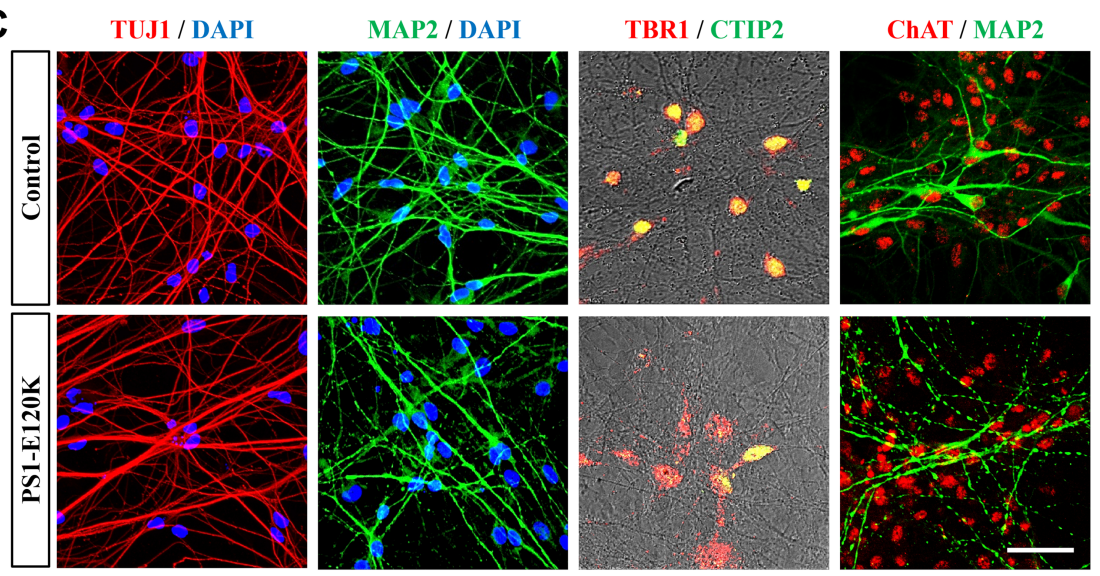

D

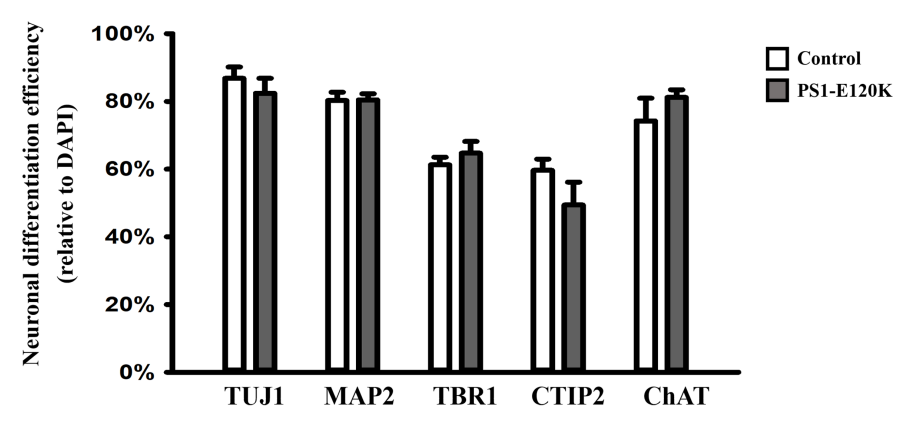

Fig. 3. Cortical neuron differentiation of PS1-E120K- and control-derived iPSCs. (A) Schematic diagram showing our stepwise cortical neuronal differentiation protocol. Control and PS1-E120K patient-derived iPSC lines were differentiated into neural precursor cells (NPC) using the Dual SMAD inhibition method. Afterwards, NPCs were treated with neurotrophic factors including BDNF, GDNF and NT3 to induce cortical neurons. (B) Immunofluorescence analysis of control and PS1-E120K iPSC-derived NPCs, showing the expression of NPC markers, such as Nestin (green), SOX2 (red) and Musashi (green) with DAPI (blue). (C) Immunofluorescence analysis of control and AD-iPSC-derived cortical neurons (TUJ1 [red] and Map2 [green]). Cholinergic neurons (ChAT [red]) and cortical neurons (TBR1 [red] and CTIP2 [green]) at 10 weeks after differentiation were shown. (D) Ratios of each mature neuronal cell type relative to DAPI staining. Scale bar: $50 \mu \mathrm{m}$. 
medium change. Strikingly, PS1-E120K iPSC-derived neurons exhibited a dramatic increase in $\mathrm{A} \beta_{42}$ levels (over 2 -fold) compared with the control iPSC-derived neurons at both 6 weeks and 10 weeks after differentiation (Fig. 4A). However, no difference in $A \beta_{40}$ levels was detected (Fig. 4B). Importantly, the ratio of $A \beta_{42} /$ $A \beta_{40}$ was significantly increased (over 2-fold) in PS1-E120K iPSC-derived neurons (Fig. 4C) compared with the control iPSCderived line. Furthermore, intracellular $A \beta_{42}$ levels and the ratio of $A \beta_{42} / A \beta_{40}$ was also significantly increased in PS1-E120K iPSCderived neurons (Fig. 4D F). We also observed a marked increase in APP expression levels in PS1-E120K iPSC-derived neurons (Fig. $4 \mathrm{H}$ and I). To detect the $\mathrm{A} \beta$ deposits, iPSC-derived neurons were stained with the anti-A $\beta$ antibody $6 \mathrm{E} 10$ and $4 \mathrm{G} 8$ at 10 weeks of differentiation. Using a confocal microscopy we found a distinctive increase of extracellular A $\beta$ deposits in PS1-E120K iPSC-derived neurons compared with the control iPSC-derived neurons (Fig. $4 \mathrm{G}, \mathrm{H})$. Given the difference in ages when the blood was drawn to generate both iPSC-derived neurons, the current findings suggest that the accumulation of $A \beta$ protein can be driven by the PS1E120K mutation beyond the effect of aging.

\section{Elevated phosphorylated-tau levels in the PS1-E120K iPSC- derived neurons}

Tau protein normally exists as a soluble form in axons. However, as it becomes hyperphosphorylated, as in $\mathrm{AD}$, it accumulates in dendrites and cell bodies $[16,17]$. To analyze the phosphorylated tau (p-tau) levels in the iPSC-derived neurons, we performed western blot analyses using antibodies against AT180 (phosphorylated at Thr231), p-Tau (phosphorylated at Ser400, Thr403/Ser404), and Tau5 (total tau). Then, we found that there exist multi-bands indicative of fractions in AT8, AT180 and p-Tau and the protein expression of each was strongly increased in the PS1-E120K iPSCderived neurons compared with the control lines (Fig. 5A F). These findings are in line with previous studies demonstrating that brain tissues from AD patients with severe Braak stages [18, 19] and neural cells with PSEN1 and APP mutation showed multiple bands of p-tau (>50 kDa) compared with the controls [20]. We also found that there was an increase of total tau (Tau5) levels, which likely correlates with a robust increase in the aggregated tau fractions [20].

As we found the increase of fragmented tau in our PS1-E120K iPSC-derived neurons at 10 weeks of differentiation, we conducted tau ELISA to detect soluble tau using conditioned medium. We found that PS1-E120K patient exhibited significant increases in soluble tau both at 6 and 10 weeks of differentiation (data not shown). To further investigate this p-tau accumulation at the cellular level, we performed immunocytochemical analysis using an antibody against AT8 and found that, unlike in the control iPSC-derived neurons, AT8 expression in the PS1-E120K iPSCderived neurons was localized to the cell bodies (Fig. 5G J). Taken together, these results show that there are high levels of p-tau (AT8) which localizes to the cell bodies in the PS1-E120K iPSC-derived neurons, compared with the control iPSC-derived neurons.

\section{Impaired mitochondria fission and fusion protein levels in the PS1-E120K iPSC-derived neurons}

Mitochondrial dysfunction is a prominent feature in $\mathrm{AD}[22$, 23]. Recent studies have reported that extracellular $A \beta$ and phosphorylated tau could be attributed to the impaired balance of mitochondrial fission and fusion $[6,24]$. To investigate whether mitochondria fission and fusion was impaired in our PS1-E120K iPSC-derived neurons, we measured the expression levels of mitochondria fission-related proteins, Drp1 (dynamin-related protein 1), p-Drp1 (for the detection of inhibitory phosphorylation of Drp1 at Ser637) and Fis1 (mitochondrial fission 1 protein); and fusion-related protein OPA1 (optic atrophy 1 gene protein), Mfn1 (membrane proteins mitofusin 1) and Mfn2 (membrane proteins mitofusin 2). Western blot analysis revealed a significant increase of Drp1 expression, a decrease of inhibitory p-Drp1 (Ser637) as well as the ratio of p-Drp1 (Ser637)/Drp1 and a slight increase of Fis1 in the PS1-E120K group compared with the control group (Fig. 6A D). Furthermore, we found that the expression of OPA1, Mfn1 and Mfn2 was dramatically reduced in PS1-E120K group when compared with the control group (Fig. 6A, E, F and G). QPCR analyses revealed dramatic increases of fission-related gene (DRP1) expression and decrease of fusion-related gene (Mfn 1) expression in the PS1-E12K iPSC-derived neurons compared with the control neurons (Fig. 6J and K). In addition, mitophagy-related proteins PINK1 and PARKIN showed dramatic increase in the PS1-E120K cells compared with the control lines (Fig. 6A, H and I). These results strongly suggest that PSEN1 mutation may result in impaired mitochondrial fission and fusion.

\section{Abnormal autophagy-related protein expression in the PS1- E120K iPSC-derived neurons}

Defective autophagy function is known also to occur in $\mathrm{AD}$ which correlates with the presence of neuritic plaques and filamentous tau $[25,26]$. It was previously shown that PS1 is necessary for the degradation of abnormal proteins using autophagylysosome system [27]. To investigate this further in our neurons, we measured the expression levels of autophagy-related proteins, including Ubiquitin (Ub), p62 (SQSTM1, sequestosome 1; cargo protein marker), Beclin1 (autophagosome membrane formationrelated protein), LC3b (light chain 3; autophagosome formation 

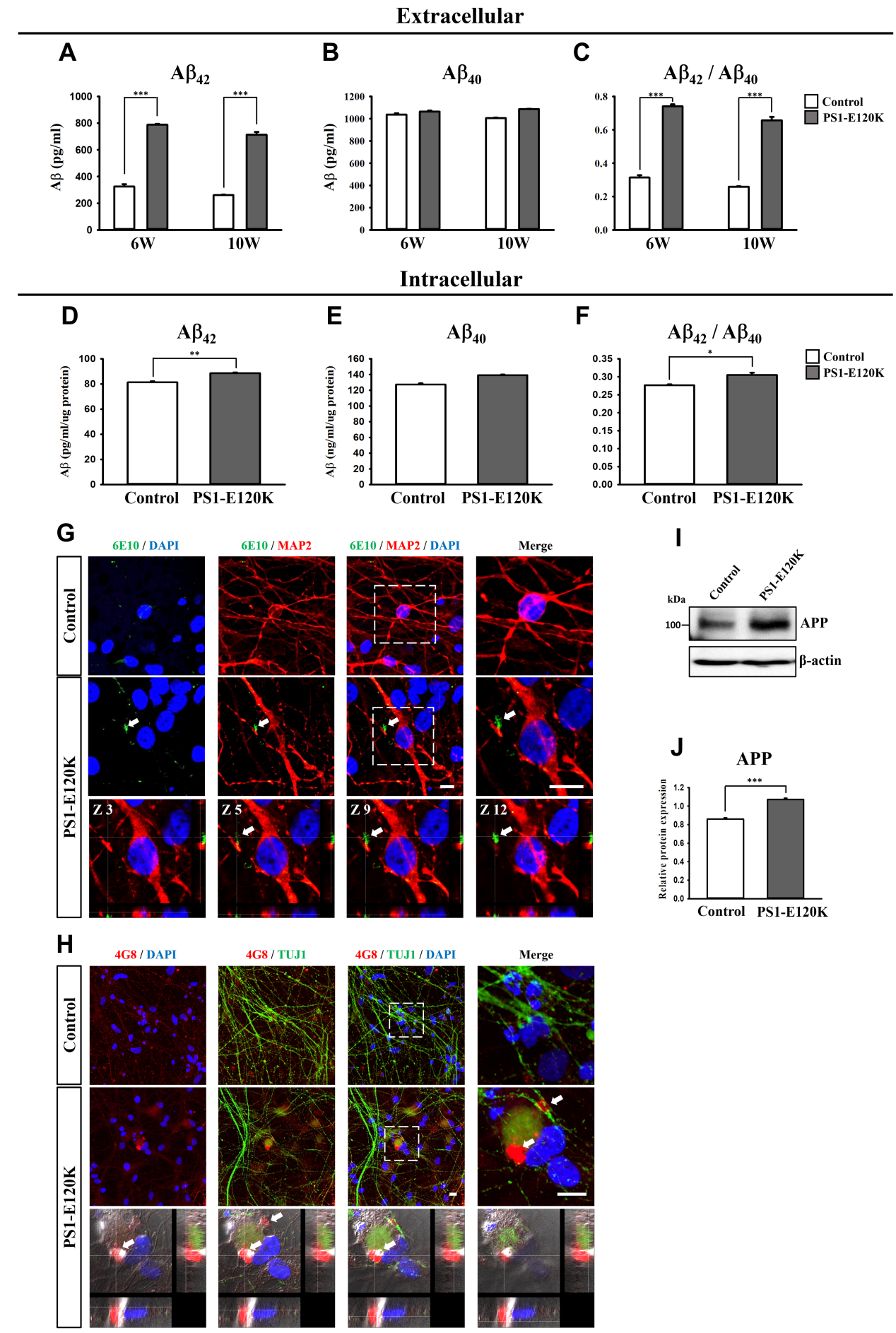

Fig. 4. Increase of A $\beta$ deposits in the PS1-E120K iPSC-derived neurons, examined at 6 and 10 weeks after neuronal differentiation. ELISA detection of $\mathrm{A} \beta_{42}(\mathrm{~A})$ and $\mathrm{A} \beta_{40}(\mathrm{~B})$ secreted from the PS1-E120K iPSC-derived neurons into the medium (extracellular), which was measured at 48 hours after the last medium change. Levels of $A \beta_{42}(A)$ and the ratio of $A \beta_{42} / A \beta_{40}(C)$ showed a dramatic increase in the PS1-E120K iPSC-derived neurons at both 6 and 10 weeks of neuronal differentiation. Intracellular $A \beta_{42}(D)$ and $A \beta_{40}(E)$ levels were measured in a total of $1 \mu g$ proteins from 10 week-differentiated neurons. Levels of the intracellular $A \beta_{42}(D)$ and the ratio of $A \beta_{42} / A \beta_{40}(F)$ showed a significant increase in the PS1-E120K iPSC-derived neurons. (G, H) Detection of $A \beta$ deposits using an antibody against $6 \mathrm{E} 10$ (shown in green) co-stained with MAP2 (red) $(\mathrm{G})$ or using an antibody against 4G8 (shown in red) co-stained with and TUJ1 (green) (H) and DAPI (blue) at 10 weeks of neuronal differentiation. The bottom panels show the z-stack images of the 6E10 and 4G8-positive A $\beta$ deposits (arrows) in the PS1-E120K iPSC-derived neurons. Scale bar: $10 \mu \mathrm{m}$. (I, J) Western blot analysis showing a significant increase of total APP expression levels in the PS1-E120K iPSC-derived neurons compared to the control. Representative ELISA, western blot and ICC images were obtained from three independent experiments. 

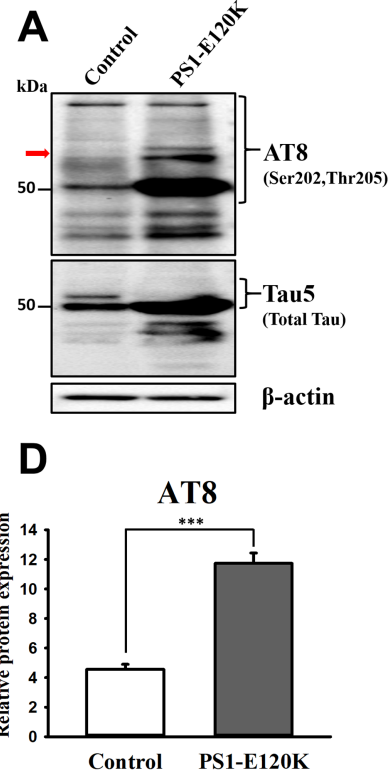

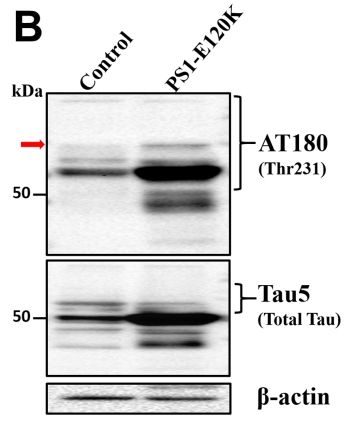

E

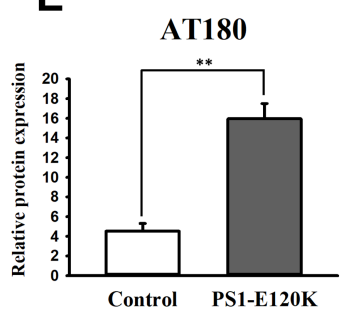

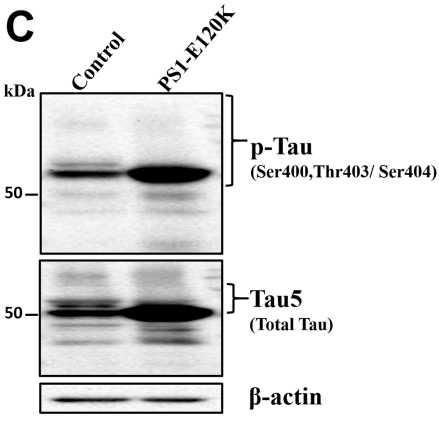

F
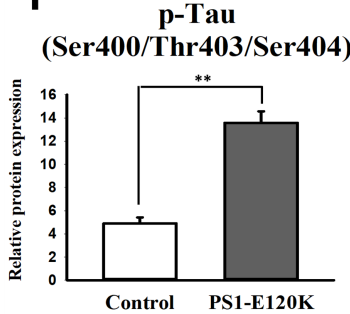

\section{G}

AT8 / DAPI
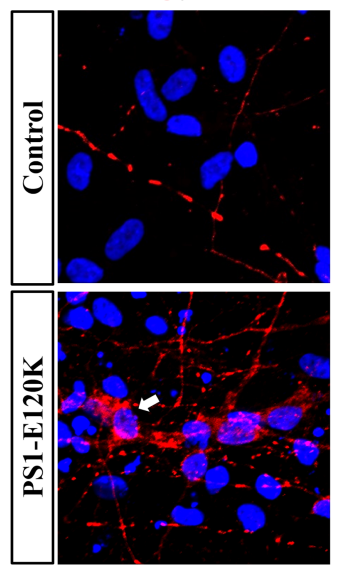

H

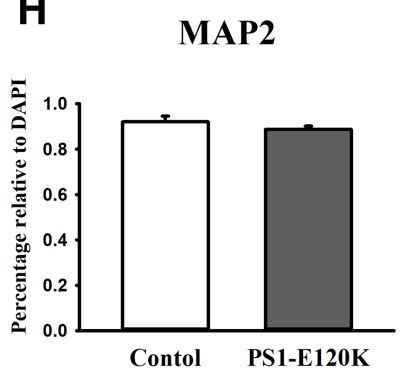

MAP2 / AT8
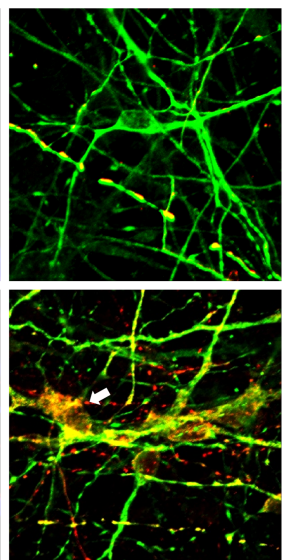

I

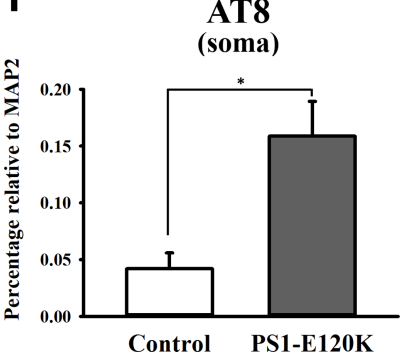

MAP2 / AT8 / DAPI
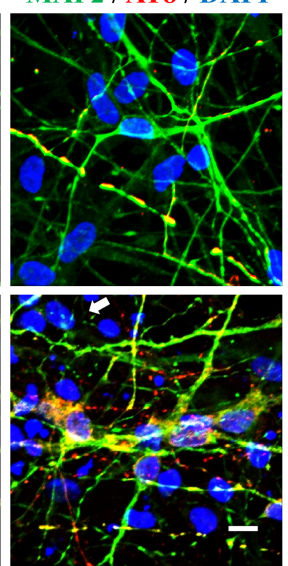

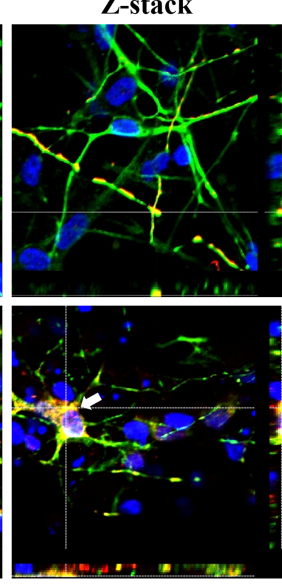

J

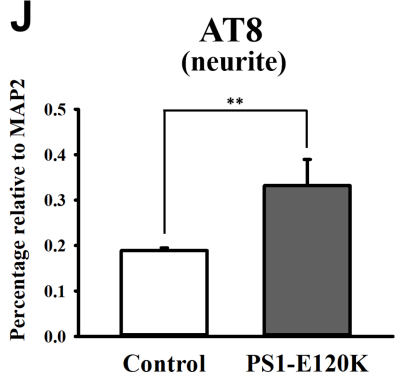

Fig. 5. Elevated p-tau levels in the PS1-E120K iPSC-derived neurons. (A C) Western blot analysis showing a significant increase of phosphorylated tau (p-tau) including AT8, AT180 and p-Tau (Ser400, Thr403/ Ser404) and total tau (t-tau, Tau5) proteins in the PS1-E120K iPSC-derived neurons compared with the control group. (D F) Quantification of the western blots on the expression of AT8 (D), AT180 (E) and p-Tau (F) showing a significant increase of the expression of each in the PS1-E120K iPSC-derived neurons. (G) Immunocytochemical analysis showing the expression of AT8 (red) and MAP2 (green), counter-stained with DAPI (blue), in the PS1-E120K iPSC-derived neurons at 10 weeks of neuronal differentiation. Note that PS1E120K iPSC-derived neurons exhibit a dramatic increase of phosphorylated-tau (AT8) both in the soma (arrow) and neurites (arrowhead) compared with the control group. Scale bar: $10 \mu \mathrm{m}$. (H J) Quantification of the immunocytochemical analysis on the expression and localization of AT8 proteins with respect to MAP2-positive neurons. MAP2 expression was normalized against DAPI (G), whereas AT8 expression was normalized against MAP2 expression (I, J). Note that significant increase of AT8 expression both in the soma (I) and neurite (J) regions in the PS1-E120K iPSC-derived neurons. Representative western blot and ICC images were obtained from three independent experiments. 

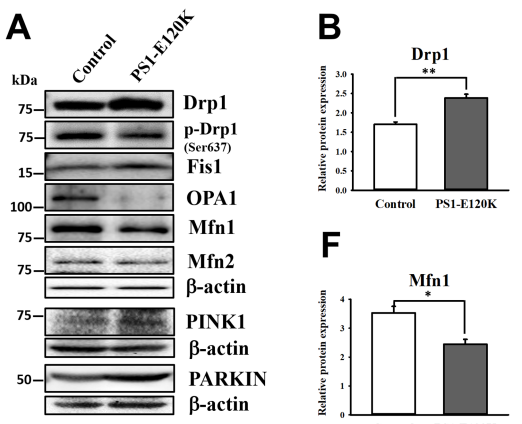

F

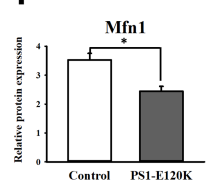

J

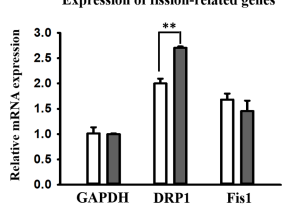

K

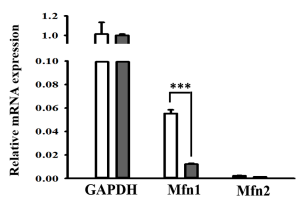

L
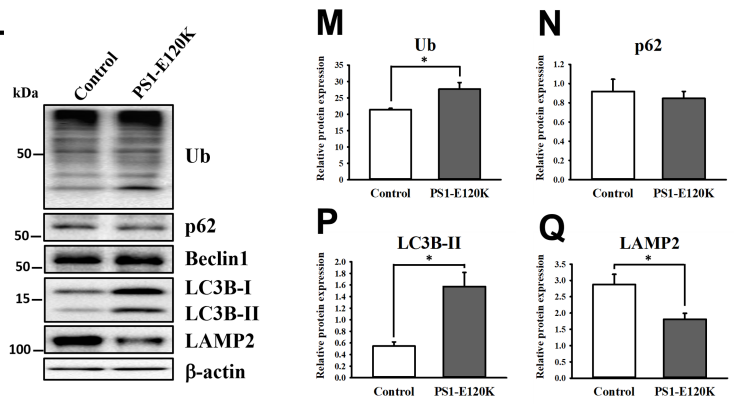

R
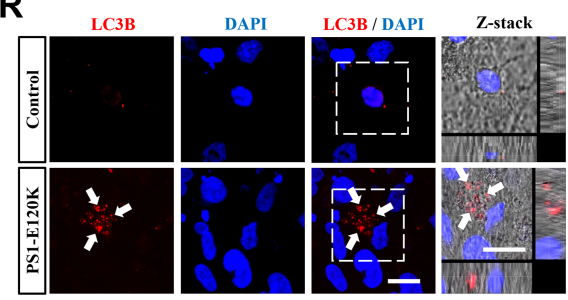

$\mathrm{S}$

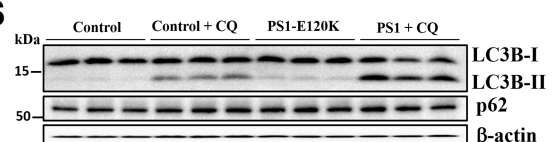

T
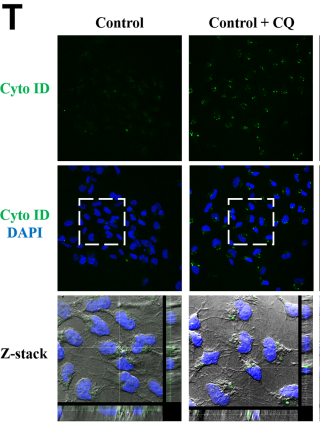

G

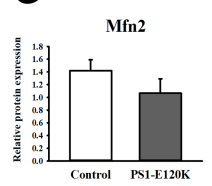

$C_{\text {p-Drp1(Ser(33)/ Drp1 }}$

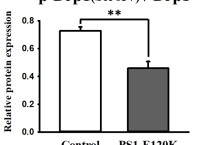

H
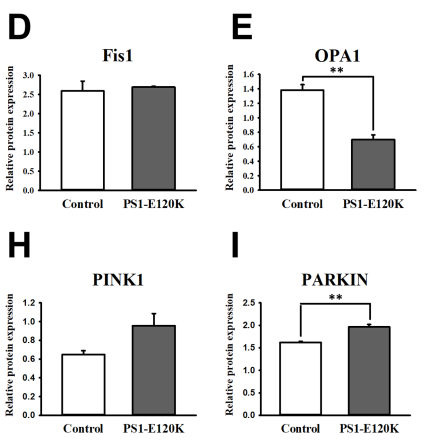

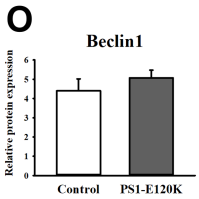

Fig. 6. Impaired balance of mitochondria fission and fusion along with defective autophagy in the PS1-E120K iPSC-derived neurons. (A) Representative western blot image from three independent experiments. (B I) Quantification of marker proteins associated with mitochondrial fission (Drpl, p-Drpl and Fis1) and fusion (OPA1, Mfnland Mfn2). Note that fission-related proteins were increased, whereas fusion-related proteins were decreased in the PS1-E120K cells compared with the control cells. Mitophagy-related protein PINK1 and PARKIN showed dramatic increases in the PS1-E120K cells compared with the control cells. (J K) QPCR analysis revealing the mRNA expression of fission and fusion-related genes in 10 weeks differentiated neurons from iPSCs. (L) Representative western blot image from three independent experiments. (M Q) Quantification of the expression of ubiquitination (Ub) and autophagyrelated proteins (p62, Beclin 1, LC3B, LAMP2). Note that levels of Ub and LC3B were significantly increased, whereas the LAMP2 signal was significantly decreased in the PS1-E120K iPSC-derived neurons. (R) Immunocytochemical staining showing the expression of LC3b (red) and DAPI (blue) in both iPSC-derived neurons at 10 weeks of neuronal differentiation. Note that PS1-E120K iPSC-derived neurons exhibit a dramatic increase of LC3b in the periphery of soma area (arrow), compared with the control group. (S) Autophagy flux assay showing a significant increase in LC3B-II expression after treatment with $10 \mathrm{uM}$ chloroquine (CQ) in the PS1-E120K iPSC-derived NPC compared with the control. (T P) CytoID (green) stained with DAPI (blue) after CQ treatment showing a dramatic increase in number of autophagic vesicles in the PS1E120K iPSC-derived NPC compared with the control. Representative western blot and ICC images were obtained from three independent experiments. Scale bar: 10 $\mu \mathrm{m}$. marker) and LAMP2 (lysosome-associated membrane proteins). Western blot analysis revealed that the expression levels of $\mathrm{Ub}$ and LC3b-II (active form of LC3b), but not p62, were significantly increased in PS1-E120K iPSC-derived neurons (Fig. 6L, M and P). To further investigate LC3B expression at the cellular level, we performed immunocytochemical analysis and found that PS1E120K iPSC-derived neurons exhibit a dramatic increase of LC3b in the periphery of soma area, compared with the control group (Fig. 6R). In contrast, the chaperone-mediated autophagosome and lysosome membrane marker, LAMP2 showed a dramatic decrease in the PS1-E120K iPSC-derived neurons (Fig. 6L and Q). To further investigate the dysfunctioning autophagy, we performed autophagy flux assay with treatment of $10 \mathrm{uM}$ chloroquine (CQ), a compound known to interfere with the lysosomal function. 
Autophagy flux assay showed a significant increase in LC3B-II expression after the CQ treatment in the PS1-E120K iPSC-derived NPC compared to the control (Fig. 6S). In addition, we performed the live cell staining with CytoID. In line with previous findings, the PS1-E120K iPSC-derived NPCs exhibited dramatic increases in the number of autophagic vesicles compared with the control cells (Fig. $6 \mathrm{~T} \sim \mathrm{P}$ ). Taken together, these results strongly suggest that PSEN1 mutation alters autophagic activity and lysosomal expression in the differentiated neurons.

\section{DISCUSSION}

To better understand AD pathogenesis, we have utilized iPSC technology and established two iPSC lines, one harboring PS1E120K mutation and one from a normal control. We did not use an isogenic control for two reasons; (i) the technical difficulty of doing this in the context of this specific mutation and (ii) the desire to look at aged control cells to exclude aging effects as the main pathological component of any disease pathogenic pathway that we identified in our mutation cell line.

We found that neurons so derived from the mutant cell line had the following characteristics. First, cortical neurons expressing $\mathrm{TBR}^{+}$and $\mathrm{CTIP}_{2}^{+}$and cholinergic neurons expressing $\mathrm{ChAT}^{+}$ were differentiated from both iPSC lines with no obvious differences in the differentiation propensity of the cell lines, nor any major morphological differences in the neurons so derived from. Second, the PS1-E120K iPSC-derived neurons exhibited higher levels of $A \beta_{42}$, an altered $A \beta_{42} / A \beta_{40}$ ratio, and $A \beta$ deposition compared with the iPSC-derived neurons developed from a normal elderly subject. Third, the levels of phosphorylated tau and its deposition in dendrites and cell bodies was seen only in the PS1E120K iPSC-derived neurons. In line with previous studies demonstrated that increased $A \beta 42 / 40$ ratio can lead increase in levels of $\mathrm{p}$-Tau through GSK3 $\beta$ activation in AD patient iPSC-derived neurons [20,21], we deduced that PS1-E120K mutation can result in increased $A \beta 42 / 40$ ratio and subsequent tau phosphorylation through the GSK3 $\beta$ activation. We have observed the same findings in another iPSC line generated from a patient carrying a different PSEN1 mutation (S170F), which exhibited a very similar pathophysiology as in the PS1-E120K iPSC line (data not shown). This new line also exhibited a very similar AD-related phenotypes, including increased levels of $A \beta$ and $p$-Tau (AT8); impaired balance of mitochondrial fission and fusion; and abnormal expression of autophagy-related proteins. Given that similar AD pathophysiology can be observed from two independent PSEN1 iPSC lines, it will be likely that the results obtained from PS1-E120K iPSC line can truly represent the pathophysiology of AD patients carrying PSEN1 mutations.

There have been several recent studies on the establishment and characterization of PSEN mutant iPSC lines, but they are mainly focused on the increase of $A \beta$ and Tau $[26,28]$. However, the present study investigated on not only $A \beta$ and Tau levels but also mitochondria and autophagy defects. Mitochondria is a highly dynamic organelle and the intracellular population at any one time derives from a balance between fission and fusion [22]. Western blot analysis revealed that fission associated markers were increased, whereas fusion related markers were markedly decreased in PS1-E120K iPSC-derived neurons compared with the control iPSC-derived neurons. As extracellular A $\beta$ and phosphorylated tau are implicated in mitochondrial fission and fusion in humans $[8,24]$, we speculated that the increased secretion and aggregation of $A \beta$ and phosphorylated forms of tau protein caused this imbalance of mitochondrial fission and fusion. Because mitochondrial fission is associated with autophagy and the genesis and degradation of autophagosomes [29], we thought the increase in mitochondrial fission may affect the clearance of pathologic protein accumulation through this pathway and this is what we found. Finally, given that the iPSC lines we generated were from a young donor, the changes in function of mitochondira and autophagy that we see must relate to the mutation and not an old cell.

There are many reports suggesting the possibility of defective autophagy in AD. It has been demonstrated that autophagy vacuoles (AVs) accumulate in human AD brains and are related to the presence of neuritic plaques and filamentous tau $[25,28]$. Furthermore, PS1 knockout mice showed a dysfunction of autophagy-lysosome system, including increase of LC3B and decrease of LAMP2 [27]. In this study, we observed significantly increased $\mathrm{Ub}$ and active forms of LC3b-II in the PS1-E120K iPSC-derived neurons, which strongly suggests that PS1-E120K mutation can induce impaired function of lysosome and the subsequent accumulation of AVs. In addition to the role of overproduction of A $\beta$ through APP cleavage, PSEN1 mutations have an important function as a chaperone for the vacuolar-ATPase to acidify the lysosomal lumen [26, 28]. We showed that the expression of LAMP2, a Chaperone-mediated autophagosome and lysosomal membrane marker, was dramatically decreased in the PS1-E120K iPSC-derived neurons. These results point towards their being abnormal autophagy activity and altered lysosomal expression in patients with PSEN1 mutation.

It should be stressed that there are several limitations to our study. First, the pateint and the elder sister of the patient had cerebellar atrophy and cerebellar symptoms and signs. Nevertheless, we were not able to further differentiate iPSC-derived neurons into Purkinje cells to evaluate relevant changes. Second, although the patient has a family history of dementia and cerebellar ataxia, 
the pedigree did not fit to an autosomal dominant patterns of inheritance. Thus, the patient may have a de novo mutation of PS1-E120K. Third, the normal elderly subject was older than the proband who provided the blood sample for the PS1-E120K iPSC generation, although this was deliberate for the reasons already stated and also explains why we did not use an isogenic control. Subsequent studies with iPSCs obtained from subjects with similar ages and the same gender as well as from mutation carriers and non-carriers in the same family will provide more thorough information of the autosomal dominant AD iPSCs. Finally, we did not perform experiments to prove any causal relationship between $\mathrm{A} \beta /$ tau formation and the dysfunction we found in mitochondria and autophagy. We observed changes of some autophagy-related proteins, such as LC3B and LAMP2, but no significant changes of p62 expression were observed.

In summary, we have shown that a PSEN1 patient with an E120K mutation has major abnormalities in terms of the iPSC-derived neurons so derived from them. These abnormalities include all the typical pathological findings of AD such as increased $A \beta$ and tau, as well as impaired mitochondrial function and abnormal autophagy activity. As such our approach will provide unique insights into the pathogenesis of autosomal dominant forms of $\mathrm{AD}$ and with this the possibility of developing new therapies.

\section{ACKNOWLEDGEMENTS}

This work was supported by the Korea Health Technology R\&D Project through the Korea Health Industry Development Institute (KHIDI), funded by the Ministry of Health \& Welfare, Republic of Korea (HI14C2746) and a grant (2016-0588) from the Asan Institute for Life Sciences.

We are also grateful to Ajinomoto for providing the StemFit ${ }^{\circledR}$ medium for our iPSC research and to Drs. John C. Morris and Chang-Seok Ki for their valuable comments for the case.

The authors declare no competing financial interests.

L.L., J.H.R., D.L.N., and J.S. were responsible for the study concept and design. L.L., J.H.R., E.H.C., Y.L., S.L., M.K., W.K., J.W.C., M.M., and H.J.K. were responsible for data acquisition. R.A.B. and M.N. critically revised the manuscript. L.L., J.H.R., and J.S. performed data analysis and manuscript writing. J.S. and D.L.N. finalized the manuscript. L.L. and J.H.R. contributed equally.

\section{REFERENCES}

1. Dubois B, Hampel H, Feldman HH, Scheltens P, Aisen P, Andrieu S, Bakardjian H, Benali H, Bertram L, Blennow K, Broich K, Cavedo E, Crutch S, Dartigues JF, Duyckaerts C,
Epelbaum S, Frisoni GB, Gauthier S, Genthon R, Gouw AA, Habert MO, Holtzman DM, Kivipelto M, Lista S, Molinuevo JL, O’bryant SE, Rabinovici GD, Rowe C, Salloway S, Schneider LS, Sperling R, Teichmann M, Carrillo MC, Cummings J, Jack CR Jr (2016) Preclinical Alzheimer's disease: definition, natural history, and diagnostic criteria. Alzheimers Dement 12:292-323.

2. Holtzman DM, Goate A, Kelly J, Sperling R (2011) Mapping the road forward in Alzheimer's disease. Sci Transl Med 3:114ps48.

3. Götz J, Ittner LM (2008) Animal models of Alzheimer's disease and frontotemporal dementia. Nat Rev Neurosci 9:532544.

4. LaFerla FM, Green KN (2012) Animal models of Alzheimer disease. Cold Spring Harb Perspect Med 2:a006320.

5. Mertens J, Paquola AC, Ku M, Hatch E, Böhnke L, Ladjevardi S, McGrath S, Campbell B, Lee H, Herdy JR, Gonçalves JT, Toda T, Kim Y, Winkler J, Yao J, Hetzer MW, Gage FH (2015) Directly reprogrammed human neurons retain aging-associated transcriptomic signatures and reveal age-related nucleocytoplasmic defects. Cell Stem Cell 17:705-718.

6. McKhann GM, Knopman DS, Chertkow H, Hyman BT, Jack CR Jr, Kawas CH, Klunk WE, Koroshetz WJ, Manly JJ, Mayeux R, Mohs RC, Morris JC, Rossor MN, Scheltens P, Carrillo MC, Thies B, Weintraub S, Phelps CH (2011) The diagnosis of dementia due to Alzheimer's disease: recommendations from the National Institute on Aging-Alzheimer's Association workgroups on diagnostic guidelines for Alzheimer's disease. Alzheimers Dement 7:263-269.

7. Christensen KJ, Moye J, Armson RR, Kern TM (1992) Health screening and random recruitment for cognitive aging research. Psychol Aging 7:204-208.

8. Manczak M, Calkins MJ, Reddy PH (2011) Impaired mitochondrial dynamics and abnormal interaction of amyloid beta with mitochondrial protein Drp1 in neurons from patients with Alzheimer's disease: implications for neuronal damage. Hum Mol Genet 20:2495-2509.

9. Itoh M, Kawagoe S, Okano HJ, Nakagawa H (2016) Integration-free $\mathrm{T}$ cell-derived human induced pluripotent stem cells (iPSCs) from a patient with lymphedema-distichiasis syndrome (LDS) carrying an insertion-deletion complex mutation in the FOXC2 gene. Stem Cell Res (Amst) 16:611-613.

10. Kondo T, Asai M, Tsukita K, Kutoku Y, Ohsawa Y, Sunada Y, Imamura K, Egawa N, Yahata N, Okita K, Takahashi K, Asaka I, Aoi T, Watanabe A, Watanabe K, Kadoya C, Nakano R, Watanabe D, Maruyama K, Hori O, Hibino S, Choshi T, Nakahata T, Hioki H, Kaneko T, Naitoh M, Yoshikawa K, Yamawaki S, 
Suzuki S, Hata R, Ueno S, Seki T, Kobayashi K, Toda T, Murakami K, Irie K, Klein WL, Mori H, Asada T, Takahashi R, Iwata N, Yamanaka S, Inoue H (2013) Modeling Alzheimer's disease with iPSCs reveals stress phenotypes associated with intracellular $A \beta$ and differential drug responsiveness. Cell Stem Cell 12:487-496.

11. Rossi M, Munarriz ER, Bartesaghi S, Milanese M, Dinsdale D, Guerra-Martin MA, Bampton ET, Glynn P, Bonanno G, Knight RA, Nicotera P, Melino G (2009) Desmethylclomipramine induces the accumulation of autophagy markers by blocking autophagic flux. J Cell Sci 122:3330-3339.

12. Brooks WS, Kwok JB, Kril JJ, Broe GA, Blumbergs PC, Tannenberg AE, Lamont PJ, Hedges P, Schofield PR (2003) Alzheimer's disease with spastic paraparesis and 'cotton wool' plaques: two pedigrees with PS-1 exon 9 deletions. Brain 126:783-791.

13. Koivunen J, Verkkoniemi A, Aalto S, Paetau A, Ahonen JP, Viitanen M, Någren K, Rokka J, Haaparanta M, Kalimo H, Rinne JO (2008) PET amyloid ligand [11C]PIB uptake shows predominantly striatal increase in variant Alzheimer's disease. Brain 131:1845-1853.

14. Verkkoniemi A, Kalimo H, Paetau A, Somer M, Iwatsubo T, Hardy J, Haltia M (2001) Variant Alzheimer disease with spastic paraparesis: neuropathological phenotype. J Neuropathol Exp Neurol 60:483-492.

15. Klunk WE, Price JC, Mathis CA, Tsopelas ND, Lopresti BJ, Ziolko SK, Bi W, Hoge JA, Cohen AD, Ikonomovic MD, Saxton JA, Snitz BE, Pollen DA, Moonis M, Lippa CF, Swearer JM, Johnson KA, Rentz DM, Fischman AJ, Aizenstein HJ, DeKosky ST (2007) Amyloid deposition begins in the striatum of presenilin-1 mutation carriers from two unrelated pedigrees. J Neurosci 27:6174-6184.

16. Spillantini MG, Goedert M (2013) Tau pathology and neurodegeneration. Lancet Neurol 12:609-622.

17. Zempel H, Mandelkow E (2014) Lost after translation: missorting of Tau protein and consequences for Alzheimer disease. Trends Neurosci 37:721-732.

18. Manczak M, Reddy PH (2012) Abnormal interaction between the mitochondrial fission protein Drpl and hyperphosphorylated tau in Alzheimer's disease neurons: implica- tions for mitochondrial dysfunction and neuronal damage. Hum Mol Genet 21:2538-2547.

19. Trojanowski JQ, Lee VM (2002) The role of tau in Alzheimer's disease. Med Clin North Am 86:615-627.

20. Choi SH, Kim YH, Hebisch M, Sliwinski C, Lee S, D’Avanzo C, Chen H, Hooli B, Asselin C, Muffat J, Klee JB, Zhang C, Wainger BJ, Peitz M, Kovacs DM, Woolf CJ, Wagner SL, Tanzi RE, Kim DY (2014) A three-dimensional human neural cell culture model of Alzheimer's disease. Nature 515:274-278.

21. Ochalek A, Mihalik B, Avci HX, Chandrasekaran A, Téglási A, Bock I, Giudice ML, Táncos Z, Molnár K, László L, Nielsen JE, Holst B, Freude K, Hyttel P, Kobolák J, Dinnyés A (2017) Neurons derived from sporadic Alzheimer's disease iPSCs reveal elevated TAU hyperphosphorylation, increased amyloid levels, and GSK3B activation. Alzheimers Res Ther 9:90.

22. Burté F, Carelli V, Chinnery PF, Yu-Wai-Man P (2015) Disturbed mitochondrial dynamics and neurodegenerative disorders. Nat Rev Neurol 11:11-24.

23. Wang X, Su B, Lee HG, Li X, Perry G, Smith MA, Zhu X (2009) Impaired balance of mitochondrial fission and fusion in $\mathrm{Al}$ zheimer's disease. J Neurosci 29:9090-9103.

24. Manczak M, Reddy PH (2012) Abnormal interaction of VDAC1 with amyloid beta and phosphorylated tau causes mitochondrial dysfunction in Alzheimer's disease. Hum Mol Genet 21:5131-5146.

25. Chesser AS, Pritchard SM, Johnson GV (2013) Tau clearance mechanisms and their possible role in the pathogenesis of Alzheimer disease. Front Neurol 4:122.

26. Nixon RA, Yang DS (2012) Autophagy and neuronal cell death in neurological disorders. Cold Spring Harb Perspect Biol 4:a008839.

27. Neely KM, Green KN, LaFerla FM (2011) Presenilin is necessary for efficient proteolysis through the autophagy-lysosome system in a $\gamma$-secretase-independent manner. J Neurosci 31:2781-2791.

28. Nixon RA, Yang DS (2011) Autophagy failure in Alzheimer's disease--locating the primary defect. Neurobiol Dis 43:38-45.

29. Youle RJ, van der Bliek AM (2012) Mitochondrial fission, fusion, and stress. Science 337:1062-1065. 